\title{
Analysis on Changes of Accessibility per Period of Outdoor Spaces of Brand-name Apartment Complexes
}

\author{
KwangJin Lee1), Ri Ryu²), YongSeong Kim³)
}

\begin{abstract}
This study objectively and quantitatively analyzed the outdoor space structure of apartment complexes from the year 2000, when brand-name apartments first appeared, until today using spatial syntax. Results of this study showed that there was little difference in the change of connectivity for each period, but it was found that the axis became more complex and grew in number over time. This is due to the increase of pedestrian walkways resulting from pedestrian-first planning that has become a recent trend. Second, it was found that while overall integration of the apartment gradually decreased, integration for unit spaces of outdoor space continuously increased, and that the growth rate was especially high for neighborhood facilities. This shows that with the arrangement of each apartment building becoming diversified, the connectivity within the complex became more important, thus leading to an increase in the linkage between outdoor space elements. Furthermore, it appears that the arrangement of public facilities that used to be established around the streets were improved for residents and users. Third, it was found that common residential spaces are recently being designed in locations with good accessibility due to the gradually increasing trends of integration. This study is significant as being a reference material in the design stage of the apartment complex through spatial syntax.
\end{abstract}

Keywords: Brand Apartment, Outdoor Space, Spatial Hierarchy, Accessibility, Spatial Syntax

\section{Introduction}

\subsection{Background and Purpose of Study}

Apartments have become one of the most representative types of residence in Korea and they play a central role in housing supply. According to the 2012 housing census, apartments accounted

Received(January 30, 2019), Review Result(1st: February 18, 2019, 2nd: March 26, 2019), Accepted(June 10, 2019)

1) (Student) 02703 Dept. Architecture Design, Kookmin Univ. 77 Jeongneung-ro, Seongbuk-gu, Seoul, Korea email: seltic12@naver.com

2) (Professor) 02703 Dept. Architecture Design, Kookmin Univ. 77 Jeongneung-ro, Seongbuk-gu, Seoul, Korea email: ri23414@hanmail.net

3) (Professor, Corresponding Author) 02703 Dept. Architecture Design, Kookmin Univ. 77 Jeongneung-ro, Seongbuk-gu, Seoul, Korea

email: yongkim@kookmin.ac.kr 
for approximately $60 \%$ of all housing units, thus representing the majority[1]. The distribution of apartments, which can supply high-concentration residences in large quantities, continued to grow since the 1970s. However, after the onset of the social issue of medium to large apartment complexes being left vacant in the mid-1990s, apartments started to be branded after late 1990s during which construction companies began distributing specialized brand apartments to improve their image and awareness. Early development of brand apartments focused less on the outside space and more on various types of main building layouts and planes. Outside spaces were recognized as side elements, but with the enactment of the law called the 'planning first-development later system' in 2003, apartments began to improve in terms of quality. Furthermore, by placing fully underground parking lots with the street width autonomy policy in 2007, the outdoor space of apartment complexes became important elements for establishing the complexes. It is necessary to analyze the apartment complexes construct up to the present to evaluate and analyze the outdoor spaces of these complexes[2].

Accordingly, this study objectively and quantitatively analyzed the outdoor spatial structure of apartment complexes from the 2000s to the present when brand apartments were introduced using spatial syntax. This study aims at constructing the basic data to be used when planning for apartment complexes in the future.

\subsection{Scope and Method of Study}

In order to analyze the outside space of brand apartment complexes, this study focused on apartment complexes from the 2000s to the present, which is the period in which large brand apartment complexes were built. Also, sampling was limited to the 'Raemian' and ' $\mathrm{i}^{\mathrm{i}}$ ' brand apartments in accordance with the order of the 2018 brand values. The spatial scope included complexes constructed in the capital area of Korea, including Seoul. The scope of the complex was limited to large complexes with 1,000 to 2,000 units.

Preceding studies on apartment complex outdoor spaces primarily focused on user satisfaction, type of outdoor space, and user perception. As such preceding studies use subjective analysis methods, there are limitations in its methods. Therefore, it is difficult to propose measures or plan elements using such resources. Therefore, this study aims at utilizing spatial syntax to quantitatively and objectively analyze the spatial hierarchy to quantitatively analyze the outdoor spatial structure. 


\section{Theoretical Study}

\subsection{Understanding of Brand Apartments}

Brands are tools that simply differentiate a product from another product. Today, however, brands now elevate the value of products. With the increase in demand for qualitative levels of residential spaces, construction companies attempted to create apartment images and unique identities through various and specialized brands. Each brand placed their brand features and strengths at the forefront to create various apartments and apartment complexes[3]. There is an increasing number of design cases in which the apartment units' interiors are constructed to look more luxurious and to give a distinctive appearance to complexes, such as diversifying the building shape. More attention is also being paid to the complex landscape. Although there is a growing trend that emphasizes a public nature by specializing the outdoor space for use by the complex community, it is normally limited to the residents of the complex.

\subsection{Facilities in the Apartment Complex}

The facilities in the complex excluding the residential spaces of apartments are divided into auxiliary facilities and welfare facilities. Auxiliary facilities include the entrance ramp for which the width is determined by the number of units, management office, landscape facilities, flood prevention lamps, information signs, communication facilities, home networks, security lamps, gas supply facilities, and emergency water supply facilities. Welfare facilities include children's playgrounds, neighborhood facilities, kindergartens, residential exercise facilities, senior citizens' center, child care facilities, libraries, and community centers[4].

In this study, outdoor spaces are defined as all spaces including buildings, excluding the residential spaces in the complex, and all other spaces that are not occupied by buildings that can be accessed by residents or pedestrians. An analysis of accessibility of outdoor spaces within the complex was selected as spaces related to the residential community. Neighborhood facilities, senior citizens' center, children's playground, and resident exercise facilities were included. In addition, the main building entrance and bracing strut entrance were included. 


\subsection{Apartment Complex Planning and Types}

The elements considered when planning for apartment complexes include the traffic line, main movement plan, common space planning, and complex boundary space planning, etc. The outdoor spatial structure of apartment complexes has close relationships with main movement plans and traffic lines. The layout of main buildings can be divided into the main building configuration or layout configuration[5]. Categorization according to the configuration can be divided into the plate type and tower-type. Although the configuration of the main building can be viewed as the structure that determines the outside spatial structure of the complex, this approach has limitations[6]. The outdoor spatial structure is determined according to the layout plans of the main building and, depending on the time, the layout plans and traffic plans have changed in various ways according to social factors and trends.

\subsection{Study on Spatial Hierarchy Analysis Using Spatial Syntax}

While apartment complexes can be seen as small unit spaces within a city, they can be analyzed as a method for evaluating social logic. Spatial syntax, which was advocated by UK Professor Bill Hillier, claims that all architectural spaces have a social logic and begins with the concept that the society that includes the residential space reflects the cultural attributes[7][8]. The theory suggests a methodology for computing the social and cultural functions and hierarchy of meaning through the relative depth of each space in a quantitative and mathematical method. Accordingly, this study proposed spatial syntax as the methodology for analyzing the spatial structure of brand apartments and for assessing its level of integration, awareness, and intelligibility[9]. This study also proposed spatial syntax as the methodology for analyzing the space of brand apartment complexes and to evaluate its connectivity, integration, and intelligibility[10].

Spatial syntax is a technique for mathematically digitalizing the relationship of spaces for analysis, and based on the connectivity between spaces, it is possible to analyze the usage, control, global integration, local integration, and intelligibility of spatial structures. In this study, spatial analysis was conducted using the axial and the axial of the convex. The evaluation indices were set as connectivity, global integration, local integration, and intelligibility. The evaluation indices are as follows.

\section{Connectivity}

Connectivity is the number of specific spaces being connected to nearby unit spaces. A higher 
connectivity means that there is a high usage of space and that the respective space has a high-usage frequency. Accordingly, spaces with high usage can be expected to be spaces that can result in confusion due to many overlapping traffic lines, and can be interpreted to have an important position in the traffic line.

\section{Global Integration}

Global integration is an index that expresses the relative depth for a specific space to access a nearby unit space. A higher integration means that there is more ease of access to other spaces. Global integration is characterized by digitalizing the relationship of a specific unit space for the entire space. In apartment complexes, management offices are spaces that require high integration.

\section{Local Integration}

Local integration digitalizes accessibility of specific unit spaces instead of the relative symmetry. Normally, the convex space perceived by people are perceived as three convex spaces. Therefore, local integration deduces values according to spatial depth 3 . While global integration views the concept as access to a specific unit space from the entire spatial unit, local integration refers to the accessibility in reaching a specific unit space within the field of human cognitive abilities.

\section{Intelligibility}

Spatial structure intelligibility refers to the relationship level of connectivity and local integration. Awareness would be higher for spaces with higher connectivity. When considering the features of apartment complexes where many unspecified people live for a short period of time, the spatial structure would require high intelligibility.

\section{Brand Apartment Spatial Analysis}

\subsection{Axial Deducing and Analysis Method}

This study is an analysis on spatial hierarchy according to the outdoor space planning of brand apartment complexes and the research was conducted as follows.

First, satellite images were collected and used to identify the spatial configuration of apartment complexes. Based on this, axial maps were drawn. The axis must be segmented based on the visible areas to configure the minimal straight lines and all axial must be connected. The axial is set based on paths that can be walked on and paths connected with 
pilotis were also included.

Second, the main building was set as a single unit space excluding the entrance. The management office was deemed an independent unit space and reflected in the analysis. The reason is that the management office must be located in a place where it is conducive to control the entire space in the apartment complex.

Third, S3 Axial Analyze was used as the tool for spatial analysis, and the analysis indices are integration, connectivity, and intelligibility.

\subsection{Selection of Case Areas}

The subjects of this study were brand apartment complexes with 1,000 to 2,000 units and the space was set as the capital area of Korea, including Seoul. The subjects were divided into periods 1 to 4 and were segmented into 5-year time frames beginning from the 2000s when brand apartments became popular. The target areas are shown in [Table 1]. Axial maps were drafted for spatial analysis based on the target of analysis. The axial was drafted based on pedestrian roads.

[Table 1] Analysis Target Overview

\begin{tabular}{|c|c|c|c|}
\hline \multicolumn{2}{|c|}{ Period } & Complex & No. of Units \\
\hline \multirow{4}{*}{1} & 2003.05 & Seocho Raemian & 1129 \\
\cline { 2 - 4 } & 2003.05 & Sanghyeon Xi & 1034 \\
\cline { 2 - 4 } & 2003.06 & Jongam-dong Raemian & 1168 \\
\cline { 2 - 4 } & 2004.09 & Munjeong-dong Raemian & 1696 \\
\cline { 2 - 4 } & 2003.12 & Shinbong Xi & 1990 \\
\hline \multirow{4}{*}{2} & 2006.07 & Weolgok Raemian & 1372 \\
\cline { 2 - 4 } & 2009.09 & Yangju Xi & 1296 \\
\cline { 2 - 4 } & 2009.10 & Yeongjong Xi & 1022 \\
\cline { 2 - 4 } & 2007.07 & Osan Xi & 1060 \\
\cline { 2 - 4 } & 2005.02 & Jangan Raemian & 1786 \\
\hline \multirow{4}{*}{3} & 2010.06 & Gileum New Town Raemian & 1617 \\
\cline { 2 - 4 } & 2011.02 & Songdo Xi Harbor View & 1069 \\
\cline { 2 - 4 } & 2010.05 & Gwonseon Xi & 1502 \\
\cline { 2 - 4 } & 2012.12 & Oksua Raemian & 1821 \\
\cline { 2 - 4 } & 2010.05 & Trivera Raemian & 1330 \\
\hline \multirow{3}{*}{4} & 2015.09 & Raemian Daechi Palace & 1608 \\
\cline { 2 - 4 } & 2015.04 & Gongdeok Xi & 1165 \\
\cline { 2 - 4 } & 2016.04 & Shingeumho Xi & 1156 \\
\hline
\end{tabular}


[Table 2] Axial Analysis Results

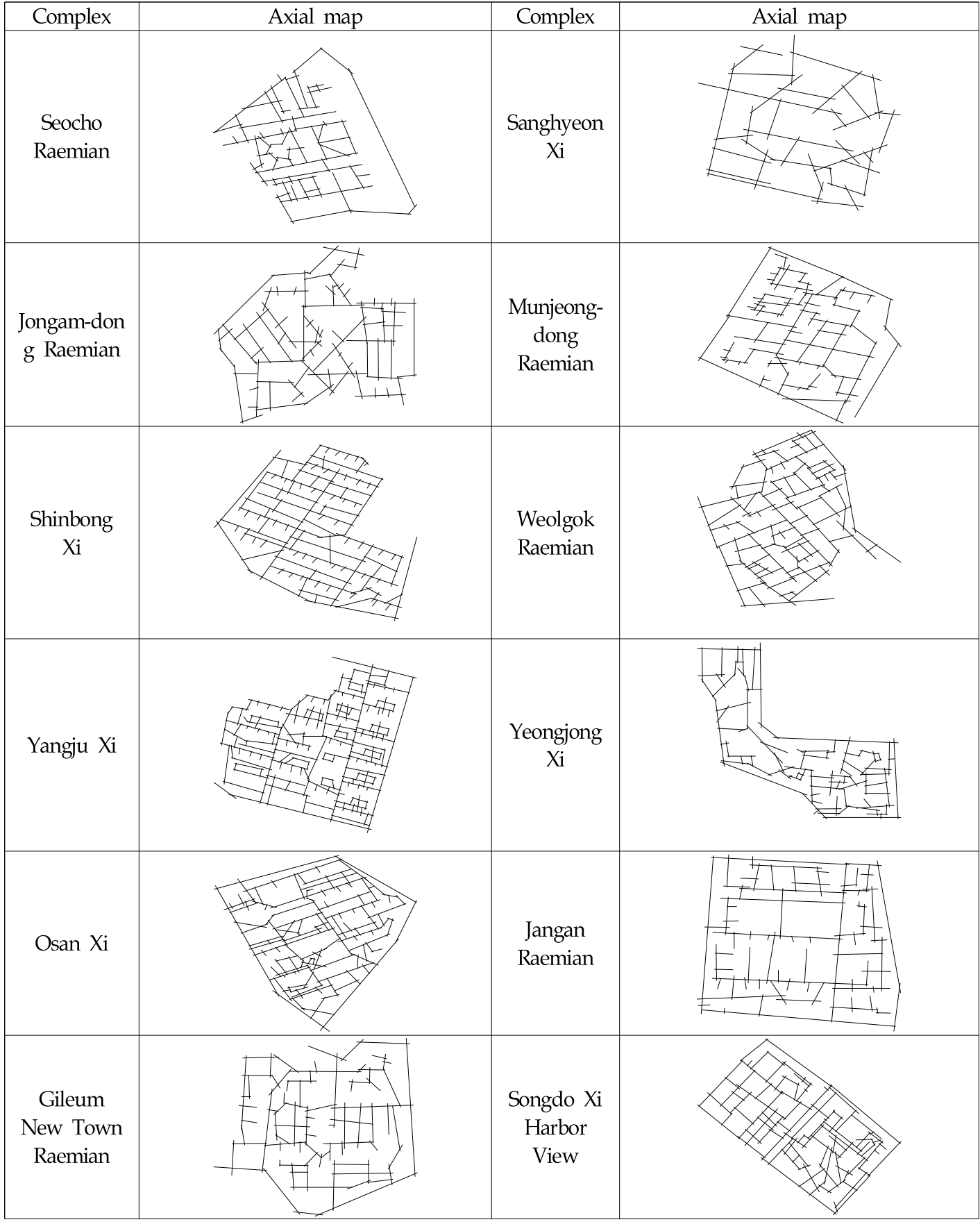




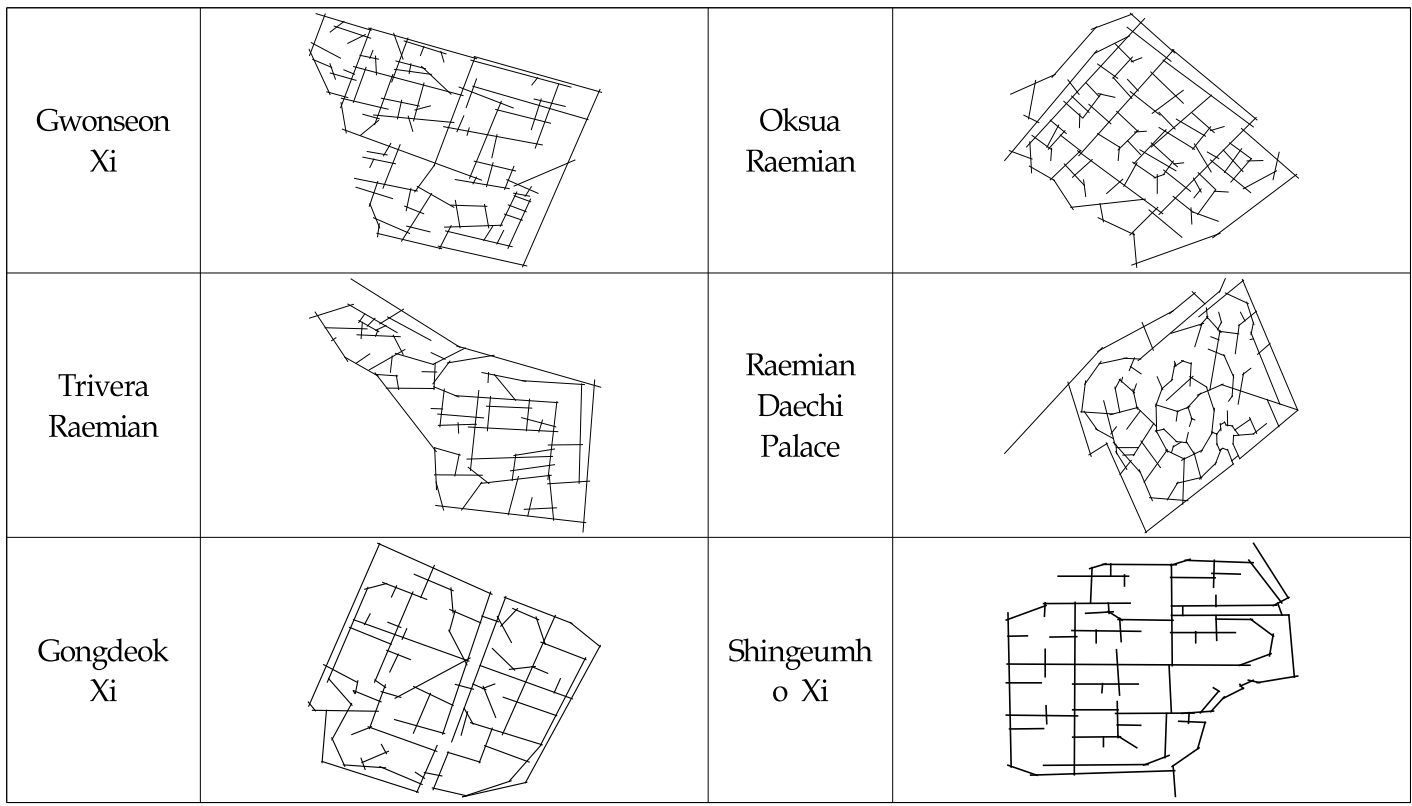

\subsection{Analysis Results and Summary}

The axial analysis results of the target complex are shown in [Table 2]. Based on the analyzed axial, the changes in the brand apartment complex and outdoor spaces were analyzed. Although the connectivity of the apartment complex outdoor space did not show significant differences, it did demonstrate a drop in global integration and local integration. This shows that the apartments in period 1 featured a layout in which the complex was the center in the overall space making it easy to perceive the spatial structure. In periods 3 and 4 , the pedestrian streets increased significantly, allowing outdoor space elements to be arranged naturally within the complex. This shows that with the diversification of layouts for each main building, connectivity within the complex became more important, thus increasing connectivity without the outdoor spatial elements. Connectivity in period 4 was the highest at 3.21, but this does not indicate that it is easy to perceive the spatial structure.

[Table 3] Apartment Spatial Structure Feature Values per Period

\begin{tabular}{|c|c|c|c|c|}
\hline & Period 1 & Period 2 & Period 3 & Period 4 \\
\hline Connectivity & 2.62 & 2.75 & 2.95 & 3.32 \\
\hline Global integration & 1.45 & 1.26 & 1.21 & 1.15 \\
\hline Local integration & 1.55 & 1.28 & 1.13 & 1.15 \\
\hline Intelligibility & 0.925 & 0.835 & 0.722 & 0.734 \\
\hline
\end{tabular}




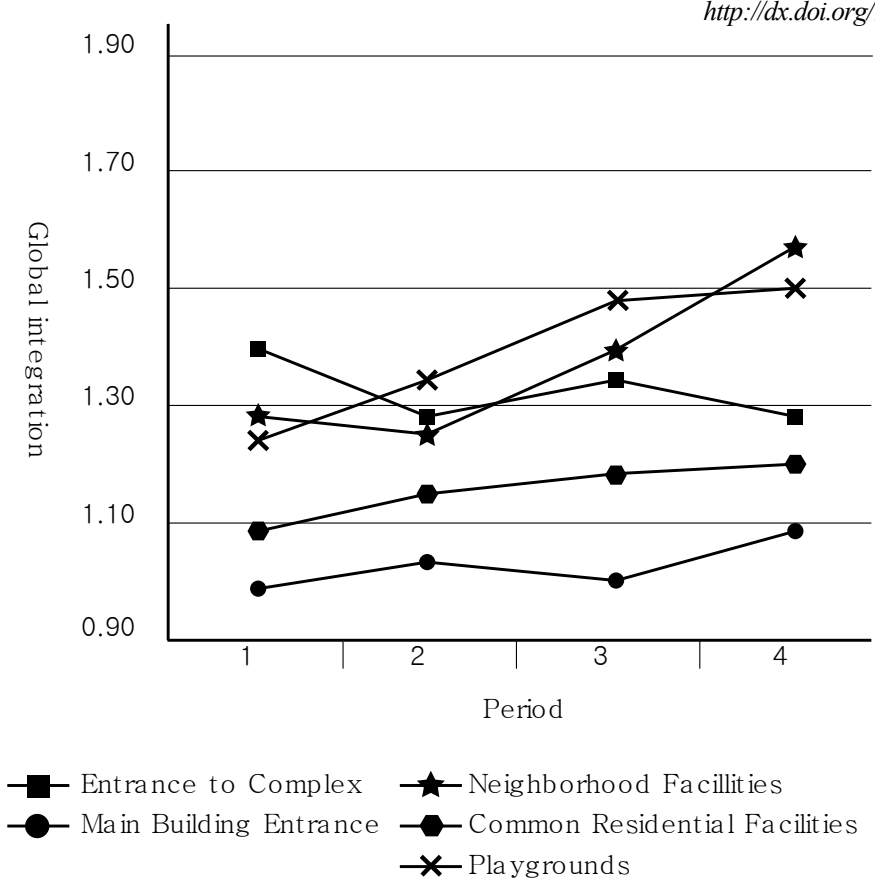

[Fig. 1] Comparison of Global Integration of Outdoor Spatial Element Spatial Structures

Excluding the entrance ramps to the complex, outdoor spaces of complexes tend to have increased in global integration. While the global integration of the entire complex decreases, the integration of outdoor spatial elements is increasing. In particular, it was found that neighborhood facilities demonstrated significant increases in integration. This means that the recent arrangement of public facilities that are centered on avenues have improved significantly in terms of residents and users.

\section{Change of Accessibility per Period for Outdoor Spaces}

\subsection{Entrance to Complex}

The mean value of integration for the entrance areas of complexes is ranked third place of all outdoor spaces. The integration value of the complex entrance area shows a small increase with the passage of time. The complex entrance at the main gate showed the highest integration in all cases. This indicates that the complex layout is made well and reflects the fact that the complex entrance, which connects the inside and outside of the complex, is where the traffic 
flow is concentrated. Meanwhile, it was found that the integration of nearby axial, excluding the complex entrance area, is low. This shows that excluding the entrance area that connects the inside and outside of the complex, a different spatial structure that is disconnected from the outdoor space was formed to create an introverted spatial structure. It can be understood that most apartments have landscaping plans or fences at its borders and are connected to the outside of the complex only through the entrance areas.

\subsection{Main Building Entrance}

In all periods, the mean value of the main building entrance had the lowest ranking. As the main building entrance is the space with the highest level of privacy that leads from the apartment complex space to the residential space, it was planned out to be the space with the lowest accessibility. Accordingly, the main building entrance space is the most segregated among the outdoor spaces. However, in recent planning cases that form the entrance by applying pilotis for the lower floors of the main building entrance, the connection from other outdoor spaces is slightly more natural. This suggests that it is apparently more advisable to plan the connected traffic line using pilotis instead of a blocked path for the main building layout while planning out the main building entrance area.

\subsection{Neighborhood Facilities}

Analysis results showed that neighborhood facilities had an increase in integration. This shows that the layouts of buildings are becoming more diversified and the connectivity within the complex has become more important. As such, the connectivity between the outdoor spatial elements is increasing. Furthermore, it is judged that the arrangement of public facilities that focus on avenues is improving for residents and users.

\subsection{Common Residential Facilities}

The integration of common residential facilities was ranked fourth place. The rankings tend to rise in more recent times and it appears that the accessibility of common residential facilities is gradually increasing. 


\subsection{Playgrounds}

The integration of children's playgrounds demonstrates similar graphs as that of neighborhood facilities. Based on periods, the integration of playgrounds was found to have continuously high values despite the changes in periods. This is judged to be attributed to the perception that when planning for complexes, playgrounds are important spaces for improving the residential community.

\section{Conclusion}

The analysis of brand apartment complex outdoor spatial structures for each period comprised hierarchical locations in the layout plans through the integration index. Relevant policies, complex planning objectives, and social issues can be substituted through this analysis to reinterpret the results. The conclusions of this analysis are as follows.

First, there was no significant change in connectivity per period. However, it was found that with the passage of time, the axial became more complex and the number of axial also increased. This appears to be the result of increased pedestrian traffic lines resulting from pedestrian-oriented planning in recent times.

Second, the integration of the entire apartment complex was found to be decreasing. In contrast, the integration of unit spaces of outdoor spaces is gradually increasing, while there was an especially high increase for neighborhood facilities. This shows that with the diversifying layout of each building and the growing importance of connectivity within the complex, the connectivity between the outdoor spatial elements is increasing. Furthermore, the arrangement of public facilities focusing on avenues appears to be improving for residents and users.

Third, in the case of playgrounds, the finding was exceptional as it maintained the integration ranking at a constant level without significant changes. It is evident that in the case of common residential spaces, the integration gradually increases and is usually allocated in locations with good accessibility.

This study has limitations as it did not exceed 20 years for the time-series analysis and that the number of cases is small. Despite this, the study is significant as it deduces and analyzes the spatial structure feature values of spatial syntax to analyze the changes and features of the outdoor spaces of brand apartments in a time-series. 


\section{Acknowledgements}

This research was supported by the National Research Foundation(NRF) grant funded by the Korea government (grant number NRF-2017R1D1A1B03029732).

\section{References}

[1] M. A. Kim, T. H. Lee and Y. U. Ban, Analysis of Urban Spatial Configuration between Seoul and Pyongyang City Using Space Syntax, Journal of the Korean Urban Management Association, (2012), Vol.25, No.1, pp.161-179.

[2] S. J. Lee and S. J. Kang, A Study on the Methodology of Positioning Security CCTV Cameras in Urban Residential District through Using Space Syntax, Journal of the Architectural of Korea Planning \& Design, (2012), Vol.28, No.9, pp.52-62.

[3] E. G. Seo, Y. J. Choi and J. P. Choi, Chronological Analysis on the Hierarchy of Service Facilities as seen in the Block Plans of Apartment Complexes, Journal of the Architectural of Korea Planning \& Design, (2014), Vol.28, No.1, pp.477-480.

[4] Y. S. Rim, The Characteristics of Qualitative Changes of Communal Space Planning in Korean Apartment Complexes, Journal of the Architectural of Korea Planning \& Design, (2011), Vol.27, No.3, pp.253-256.

[5] I. S. Park, The Trend of Space Syntax Studies and Research Problem Types, Journal of the Architectural of Korea Planning \& Design, (2015), Vol.31, No.9, pp.29-38.

[6] Y. H. Pyun, S. J. Lee and C. I. Park, A Study on the Ways of Publicness's Realization and Design Characteristics in SANAA's Museum Architecture - Focus on the analysis by space syntax, Journal of Digital Design, (2011), Vol,11, No.3, pp.433-444.

[7] J. S. Lee and H. J. Kim, A Study on the Semiotics approach to Advertisement of Brand Apartment : Focusing on Raemian and Hillstate, RESIDENTIAL ENVIRONMENT : JOURNAL OF THE RESIDENTIAL ENVIRONMENT INSTITUTE OF KOREA, (2012), Vol.10, No.3, pp.245-257.

[8] M. S. Kim and G. S. Piao, An In-depth Study on Applying Metric Weighting to Space Syntax, JOURNAL OF THE ARCHITECTURAL INSTITUTE OF KOREA Planning \& Design, (2018), Vol.34, No.12, pp.49-54.

[9] Y. S. Kim, B. U. Shin and H. K. Nam, A Study on the Plane Spatial Characteristics of Modern Hanok in the Jeonju Hanok Village using Space Syntax, JOURNAL OF THE ARCHITECTURAL INSTITUTE OF KOREA Planning \& Design, (2018), Vol.34, No.4, pp.35-44.

[10] T. S. Kim, J. M. Park, E. G. Park and D. S. Han, A Study on the Time-Sectional Analysis of Apartment Housing related research in Korea, JOURNAL OF THE ARCHITECTURAL INSTITUTE OF KOREA Planning \& Design, (2018), Vol.34, No.3, pp.45-52. 